\title{
Manejo de escenarios mediante clima y suelo en la producción de caña de azúcar (Saccharum officinarum) 2010-2020: Valle del Grullo-Autlán, Costa Sur de Jalisco
}

Management of climate and soil scenarios in the production of sugarcane (Saccharum officinarum) 2010-2020: Valle del Grullo-Autlán, Southern Coast

of Jalisco

Cenários de gerenciamento usando clima e solo na produção de cana de açúcar (Saccharum officinarum) 2010-2020 Valle del Grullo-Autlan, Jalisco

South Coast

Sandoval-Legazpi, J. de J. Departamento de Ecología, Centro Universitario de la Costa Sur, Universidad de

Guadalajara, México

slegazpi@cucsur.udg.mx

Figueroa-Rangel, B. L. Departamento de Ecología, Centro Universitario de la Costa Sur, Universidad de

Guadalajara, México bfrangel@cucsur.udg.mx

Pérez Mares, J. G. Departamento de Ecología, Centro Universitario de la Costa Sur, Universidad de

Guadalajara, México gperez@cucsur.udg.mx

\section{Resumen}

Los modelos como herramientas de simulación constituyen un medio para entender la complejidad de un determinado sistema; los modelos se consideran elementos disponibles y necesarios para entender las hipótesis en sus procesos. Un modelo permite generar situaciones reales y complejas con base en sistemas de simulación que admiten tomar decisiones de cualquier índole. Este trabajo presenta, con apoyo del software VENSIM, escenarios propicios para la producción de caña de azúcar en el Valle El Grullo-Autlán, así 
Revista Iberoamericana de las Ciencias Biológicas y Agropecuarias

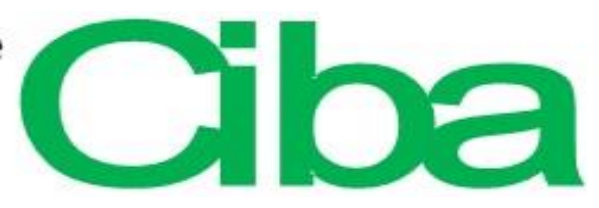

ISN : $2007-9990$

como la validación del mismo con apoyo del software SPSS (versión 15.0). Los resultados muestran la manera como los factores suelo y precipitación repercutirán directamente y a futuro en la producción de caña por hectárea en el Valle El Grullo-Autlán. En este caso, el modelo cuadrático y el cúbico fueron estadísticamente significativos para el caso de la precipitación, no así la temperatura, lo que se suma al historial de temperaturas de los últimos 14 años en la región, el cual no presenta injerencia alguna hasta el momento. La producción ton/ha para el periodo 2010-2020 reflejará un aumento estimado de entre 3 y 8 ton/ha, según estos factores. Pero también se observa que de existir excedentes en el factor lluvia y pérdidas en materia orgánica, la producción tenderá a disminuir.

Palabras clave: simulación, caña de azúcar, precipitación, temperatura, materia orgánica.

\section{Abstract}

Models as simulation tools are a means to understand and explain how complex a given system can be, since the models are considered as elements available and necessary for the understanding of hypotheses involving a series of processes within these systems. A model allows real and complex situations to be generated based on simulation systems that allow decision making of any kind. This work presents support scenarios for the production of sugarcane in the valley of El Grullo-Autlán, with the support of the software VENSIM, as well as the validation of the same with the support of SPSS software (version 15.0). The results show how the soil and precipitation factors will directly and in the future affect cane production per hectare in the El Grullo-Autlán valley. In this case the quadratic and cubic model were statistically significant for the case of precipitation, but not the temperature, which is added to the history of temperatures of the last 14 years for this region, which does not present interference so far. Ton / ha production for the period 2010-2020 will reflect an estimated increase of between 3 and 8 ton / ha. According to these factors. But it is also observed that if there are surpluses in the rainfall factor and losses in organic matter, production will tend to decrease. 
Revista Iberoamericana de las Ciencias Biológicas

y Agropecuarias

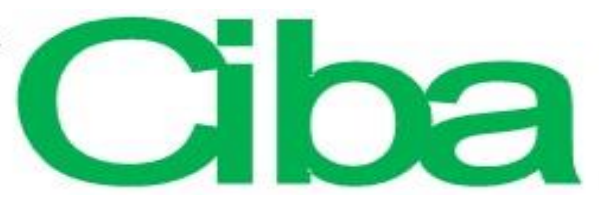

ISSN: 2007 - 9990

Key words: simulation, sugar cane, precipitation, temperature, organic matter.

\section{Resumo}

Modelos e ferramentas de simulação são um meio para compreender a complexidade de um dado sistema; elementos são considerados modelos disponíveis e necessários para compreender os pressupostos em seus processos. Um modelo pode gerar situações reais e complexas baseadas em sistemas de simulação que suportam a tomada de decisões de qualquer tipo. Este artigo apresenta, com o apoio do software VENSIM, permitindo cenários para a produção de cana no Valle El Grullo-Autlan e sua validação com o apoio do software SPSS (versão 15.0). Os resultados mostram como os factores de solo e de precipitação têm um impacto directo e o futuro na produo de cana por hectare no Valle Grullo-Autlán. Neste caso, o modelo quadrático e cúbico foram estatisticamente significativas para o caso de precipitação, não temperatura, o que aumenta a história da temperatura dos últimos 14 anos na região, que não apresentam qualquer interferência com a tempo. Produção toneladas / ha para o período de 2010-2020 irá reflectir um aumento estimado de entre 3 e 8 ton / ha, de acordo com estes factores. Mas também observa que o excedente existe no fator de chuva e perda de matéria orgânica, a produção tende a diminuir.

Palavras-chave: simulação, cana de açúcar, precipitação, temperatura, matéria orgânica.

Fecha recepción: Octubre 2016

Fecha aceptación: Mayo 2017

\section{Introducción}

El municipio de Autlán cuenta con una gran actividad agrícola, en la que predomina el cultivo de azúcar en grandes extensiones de tierra, y en menor medida el cultivo de maíz y hortalizas. La alta producción de azúcar resultado de la cantidad de tierra cultivada con caña de azúcar, convierte al Valle El Grullo-Autlán en uno de los más productivos del país (CONADESUCA, 2015). 
Revista Iberoamericana de las Ciencias Biológicas y Agropecuarias

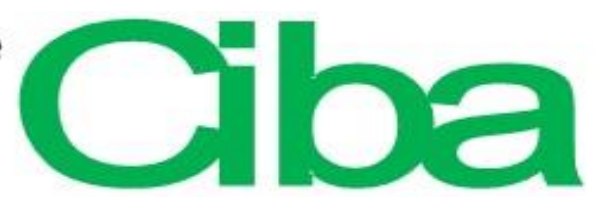

ISSN: $2007-9990$

Cabe mencionar que dicha producción es amenazada cada año por la disminución de nutrientes del suelo, que junto con el clima influye en el desarrollo y crecimiento del cultivo. Además, en los últimos años ha habido escasa lluvia, ocasionando que la producción sea sumamente variable (CONAGUA, 2014).

En el Valle El Grullo-Autlán es necesario tomar acciones radicales con respecto al manejo adecuado de la tierra y el agua - de riego y de lluvia-, ya que cada año empeora la situación.

De ahí la necesidad de planear y prever las condiciones que puedan presentarse en los próximos diez años. La finalidad de este trabajo es generar estrategias que ayuden a analizar los factores que afectan la producción de caña de azúcar, por ejemplo, el clima y el edáfico. De esa manera, se apoya al sector productivo organizado de la región: Confederación Nacional de Propiedad Rural (CNPR), Confederación Nacional Campesina (CNC) e Ingenio Melchor Ocampo (IMO), para que se beneficie junto con los demás actores involucrados.

Las necesidades de los productores de caña de azúcar, como el manejo de suelos y la producción de caña de azúcar, pueden satisfacerse mediante el simulacro de escenarios.

\section{Revisión de literatura}

La simulación es una forma efectiva de estudiar los comportamientos dados en una cadena de suministro, así como un camino práctico para entender cómo funciona un sistema complejo, donde participan diferentes actores sujetos a variables interrelacionadas, las cuales definen un comportamiento base de acuerdo a los factores utilizados (Liu et al., 2004).

Por otra parte, la base de un modelo construido a partir de la dinámica de sistema es una hipotética explicación básica, pero completa, de un sistema real, el cual es capaz de reproducir los comportamientos de entradas y salidas de datos (Robinson, 2006, citado en 
Revista Iberoamericana de las Ciencias Biológicas

y Agropecuarias

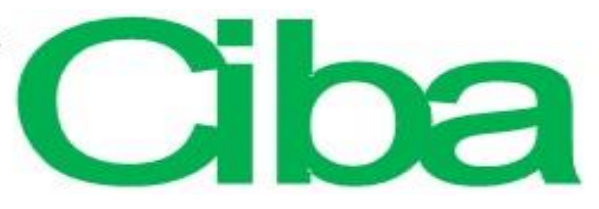

ISSN: 2007 - 9990

Vergara, 2008; Keenan, 2010; García, 2011). La aplicación de la dinámica de sistemas en el ámbito agrícola ha estado presente en prácticamente los últimos 60 años.

\section{La aplicación de dinámicas de sistemas en el ámbito agrícola}

Como parte de la producción de forrajes, Holmann (2002) mostró los beneficios que se pueden obtener del uso de modelos de simulación utilizando como estudio de caso la cuantificación del impacto de nuevas alternativas forrajeras sobre el costo de producción de leche y sus implicaciones para la adopción tecnológica y la sostenibilidad del uso de la tierra en Costa Rica y el Perú.

Asimismo, estudios realizados por Vergara (2008) en la ciudad de Bolívar en Colombia manejaron esta herramienta y mostraron la utilización de las redes dinámicas en las cadenas productivas agroindustriales, como complementaria para el estudio a partir de la evaluación de escenarios posibles que apoyaran el desarrollo regional.

También en Colombia se presentó un trabajo de simulación de escenarios, lo que permitió identificar alternativas probables de manejo en el rubro hortofrutícola. En este estudio se resume un arduo trabajo de modelado de las cadenas productivas hortofrutícolas más importantes del departamento de Bolívar mediante la simulación de redes (Fontalvo et al., 2009).

Con respecto a los cultivos, Hernández et al. (2009) revisaron los diferentes modelos de simulación para la generación de escenarios, tomándolos como una herramienta útil en los procesos de toma de decisiones, para poder lograr posteriores aplicaciones como primera aproximación de la capacidad productiva en distintas condiciones edafoclimáticas. Sus conclusiones fueron que estos modelos constituyen una herramienta muy útil para poder desarrollar una agricultura eficiente, desde el punto de vista económico, sin embargo, tal vez es más importante hacer un uso racional de los recursos naturales, teniendo en cuenta la conservación del medio ambiente y, sobre todo, del recurso suelo. 
Revista Iberoamericana de las Ciencias Biológicas

y Agropecuarias

Igualmente, en la parte agrícola, Nicholson (2005), citado por Keenan, (2010), sugirió que la modelación de la dinámica de sistemas podría ser un mecanismo eficaz para la evaluación de problemas complejos en contextos de desarrollo rural internacional.

Además, en lo que respecta a la simulación en caña de azúcar, Aguilar et al. (2010) mencionan el manejo de escenarios desde una perspectiva general y no como manejo de software, es decir, proponen que, de acuerdo a la calidad y cantidad de cada uno de los ingenios existentes en México, se debería hacer más eficiente la producción de cada uno de ellos, sobre todo cuando la tendencia es la producción de biocombustibles a través de ese y otros cultivos.

Dichos autores también señalan que los ingenios de baja producción deberían ser integrados a la producción de biocombustibles, los de media producción a la del azúcar y los biocombustibles, y los de mejor calidad únicamente a la producción de azúcar. Esta es una propuesta razonable tomando en cuenta la demanda mundial de biocombustibles y la escasez de hidrocarburos ocasionada por el agotamiento de los yacimientos petroleros.

La Ingeniería de Sistemas es una de las herramientas más útiles en la generación de escenarios y en la toma de decisiones. Así lo señalan Fontalvo et al. (2010) cuando exponen que la simulación permite experimentar con un modelo, que es una versión simplificada de un sistema real. Cabe aclarar que la simulación no es una herramienta de pronóstico, sino más bien una para la creación y validación de escenarios.

En este caso, se presentan factores físicos como el clima y el suelo, los cuales influyen directamente en la producción de caña de azúcar. Dicho factor climático (precipitación) afecta directamente a la variable suelo, donde los valores de textura, $\mathrm{pH}$ y materia orgánica rigen en gran medida su fertilidad. 
Revista Iberoamericana de las Ciencias Biológicas y Agropecuarias

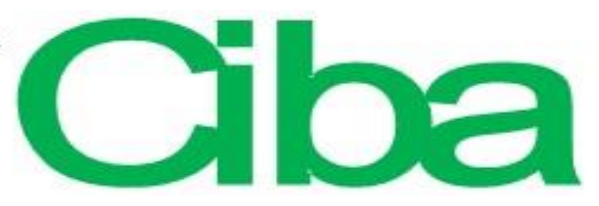

ISSN: $2007-9990$

El escenario observado es reciente en cuanto a clima y suelo y propone acciones para un mejor manejo del cultivo de la caña de azúcar para el periodo 2010-2020, con apoyo del software VENSIM, utilizando las variables de clima (precipitación y temperatura) y suelo (textura, pH y materia orgánica), y su relación con la producción de caña de azúcar (ton/ha). Asimismo, presenta la validación del modelo mediante el software SPSS (versión 15.0).

\section{Materiales y métodos}

El Valle El Grullo-Autlán, ubicado en los municipios El Grullo y Autlán (19³5’ a $19^{\circ} 54$ de latitud Norte y $104^{\circ} 07^{\prime}$ a $104^{\circ} 29^{\prime}$ de longitud Oeste (INEGI, 2000) (figura 1), tiene un clima principalmente semiseco con precipitaciones medias de $900 \mathrm{~mm}$ al año y temperaturas medias de $24^{\circ} \mathrm{C}$ y máximas de más de $32^{\circ} \mathrm{C}$ (SEI-JAL, 2000). Los suelos son del tipo Feozem haplico, Fluviosol eutrico, Vertisol pélico y Regosoles en las partes altas y pie de monte (SPP, 1981).

Figura 1. Ubicación del Valle El Grullo-Autlán (Costa Sur de Jalisco).

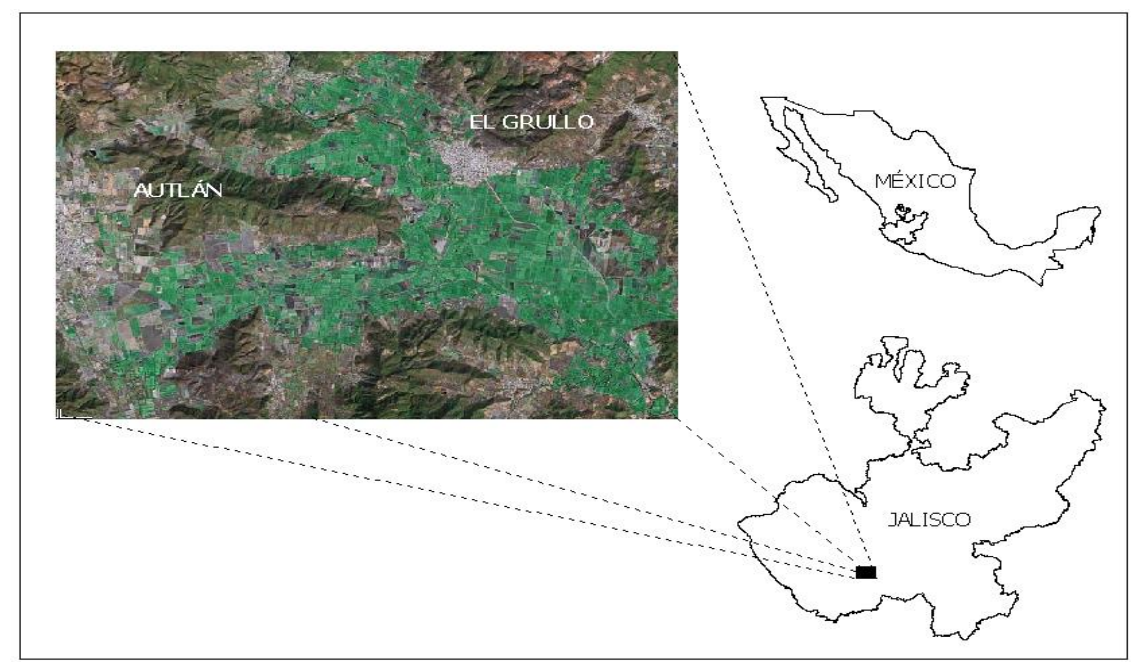

Fuente: Google Earth, 2014 
Revista Iberoamericana de las Ciencias Biológicas y Agropecuarias

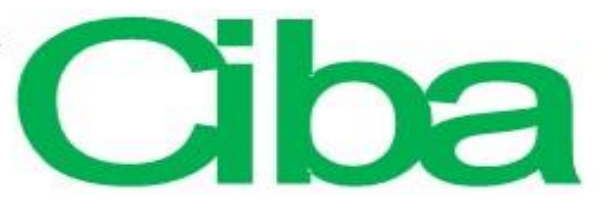

ISSN: 2007 - 9990

Para el estudio asimismo se utilizaron datos promedio georreferenciados de textura, materia orgánica, y pH de un proyecto de suelos, así como información de producción de caña de azúcar (ton/ha), generada por el Ingenio Melchor Ocampo (IMO), y de precipitación (mm) y temperatura de 14 años (2001-2014) de la estación climatológica El Chante (la más completa), con los que se alimentó el software VENSIM (figura 2).

La importancia de estas tres variables es ampliamente reconocida, así lo señalan varios autores (Acevedo et al., 2010; Ferreras, et al., 2007; Lesur, 2006; Castellanos et al, 2000; y Ortíz y Ortíz, 1990), quienes mencionan que dichas variables son de gran riqueza nutrimental en las parcelas e inciden de manera potencial en la fertilidad de los suelos.

La información de datos para la alimentación del modelo se dio bajo el siguiente contexto: la producción nacional y regional de la caña de azúcar es variable y depende de las necesidades (demanda) de la población y del stock presente en las bodegas de almacenamiento, lo que genera la oferta hacia la población. 


\section{Revista Iberoamericana de las Ciencias Biológicas y Agropecuarias}

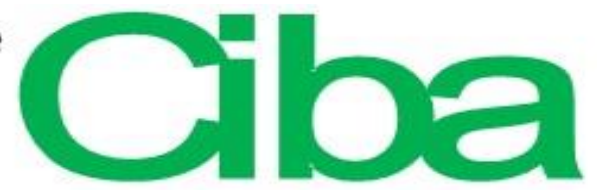

. Figura 2. Software y desplegado de variables utilizadas para la generación de escenarios en la producción de caña de azúcar (ton/ha.) en el Valle El Grullo-Autlán.

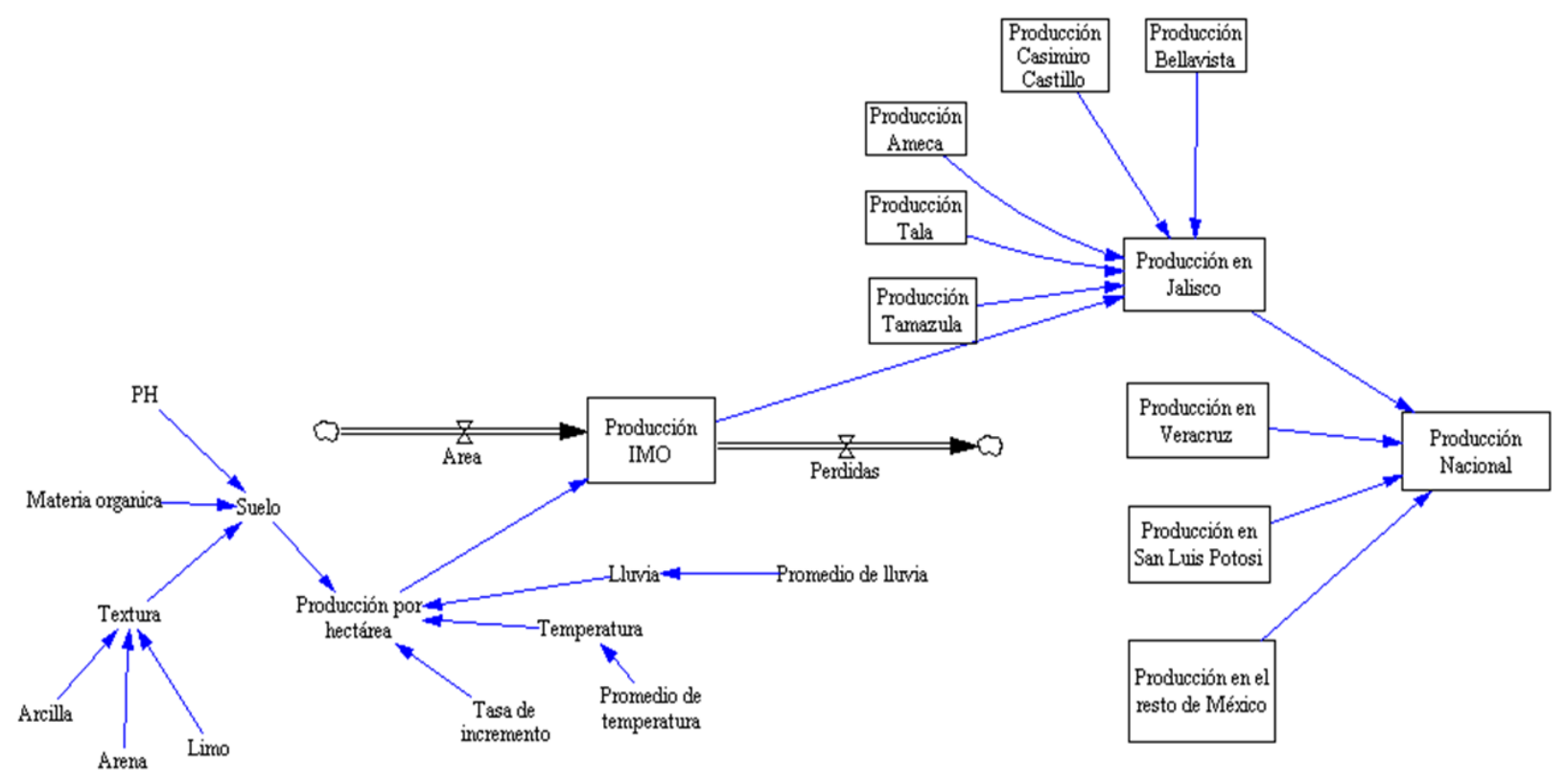

A nivel nacional y local, en lo que respecta al Valle El Grullo-Autlán, la producción depende de las pautas que señalan las necesidades nacionales con base en un estimado para cada ingenio cañero y donde el tope de producción lo pone precisamente esa necesidad nacional, con lo que se determina el número de hectáreas a sembrar con un estimativo promedio de producción de caña de azúcar/hectárea/ingenio/año.

Con base en lo anterior se tomaron los siguientes valores reportados para el Valle El Grullo-Autlán (hasta 2011): $:^{1,2}$.

${ }^{2}$ USDA (2011). Word production, supply, and distribution, centrifugal sugar. Sugar and Sweetener Yearbook, 2011. United States of America Department Agricultural.

${ }^{2}$ UNC (2011). México en el mundo. Unión Nacional de Cañeros, A. C.-CNPR. En: http://www.caneros.org.mx/principal.html\# 
Revista Iberoamericana de las Ciencias Biológicas y Agropecuarias

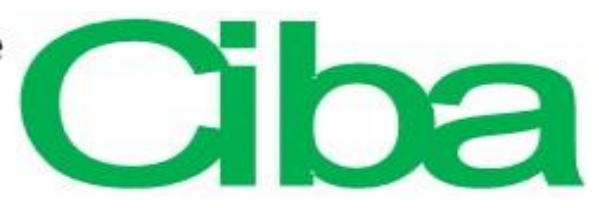

ISSN: 2007 - 9990

La producción mundial de caña de azúcar fue de 160948 ton/año (stock mundial), mientras que la producción nacional de este mismo producto fue de 4652 ton/año (stock nacional). La producción promedio para el Valle El Grullo-Autlán fue de: 94.21 ton/ha (zafra 20102011). Exportada: 690 ton/año. Importada: 900 ton/año. Caña de azúcar disponible para México: producción total menos la cantidad exportada más la cantidad importada: 4862 ton / año. Producción de caña de azúcar para Veracruz: 2052 ton/año. Producción de caña de azúcar para Jalisco: 573 ton/año. Producción de caña de azúcar para San Luis Potosí: 378 ton/año. Producción de caña de azúcar para el resto de los estados: 2376 ton/año. Precipitación promedio: $800 \mathrm{~mm} / \mathrm{año}$. Temperatura promedio: $28^{\circ} \mathrm{C}$. Textura promedio: franca arenosa. PH promedio: 6-7. Materia orgánica promedio: $1 \%$.

En cuanto a los datos, se utilizaron los valores de producción de los últimos 14 años tomados de la página web de la Unión Nacional de Cañeros, A.C. CNPR, así como datos de precipitación y temperatura proporcionados por la Comisión Nacional del Agua (CONAGUA, 2014), generados en la Estación Climatológica "El Chante", en la localidad del Municipio Autlán de Navarro (los más completos que se tenían de este valle).

\section{Resultados y discusión}

1) Software VENSIM:

a) Variable suelo: Considerando las anteriores variables promedio, la textura, el $\mathrm{pH}$, la materia orgánica y la precipitación (lluvia), se tiene que con un "look up" cuyo valor es uno (1), como dato promedio base para el cultivo de la caña de azúcar las variables edáficas serán constantes.

b) Variable precipitación $(\mathrm{mm})$. Para esta variable el comportamiento fue similar al suelo. Esto es que mientras el "look up" se mantenga en 1, también estas variables serán constantes. 
Revista Iberoamericana de las Ciencias Biológicas

y Agropecuarias

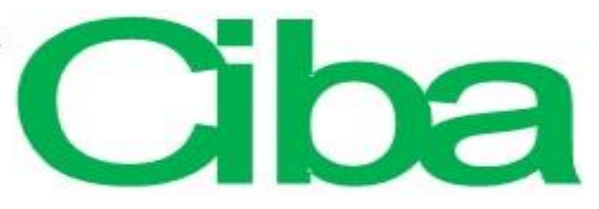

ISSN: 2007 - 9990

c) Variable temperatura $\left({ }^{\circ} \mathrm{C}\right)$. Para el caso de la temperatura, el comportamiento fue similar que la variable suelo y precipitación, aunque aparentemente dicha variable no tuvo mucha injerencia en la producción de caña de azúcar por hectárea.

d) Producción ton/ha en el Valle El Grullo-Autlán. Aunado a lo anterior y como parte de los valores actuales de producción de caña de azúcar en ton/ha, se tiene que con valores hasta el 2013 de una producción promedio de caña de azúcar de 94 ton/ha y con valores promedio de suelo en cuanto a textura franco arenosa, $\mathrm{pH}$ de 6.5 y materia orgánica de $1 \%$, la simulación a 10 años (2010-2020) sería como sigue (Gráfica 1).

Gráfica 1. Proyección de la producción toneladas por hectárea de la caña de azúcar (ton/ha) en el Valle El Grullo-Autlán para el periodo 2010-2020.

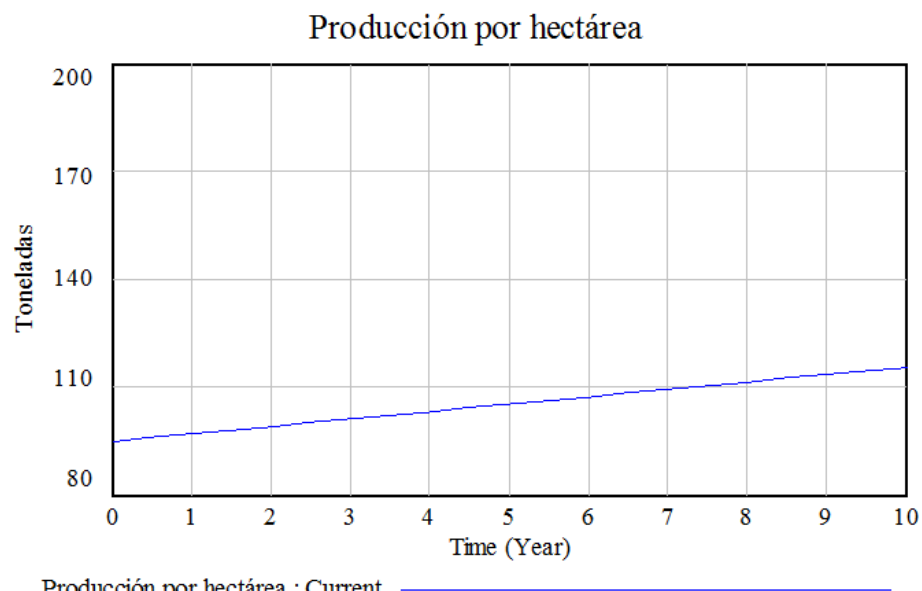

En la Gráfica 1 se puede observar la tendencia al aumento gradual de aproximadamente 2 toneladas por año, esto es, poco más de veinte toneladas adicionales por hectárea de producción de caña de azúcar para el año 2020 (producción promedio a los próximos 10 años, la cual puede presentar ligeras variaciones en este periodo), si los valores de suelo (textura, $\mathrm{pH}$ y materia orgánica) y clima (precipitación y temperatura), se mantienen o ligeramente mejoran, como en el caso de la materia orgánica con la adición de abonos orgánicos. 
Revista Iberoamericana de las Ciencias Biológicas

y Agropecuarias

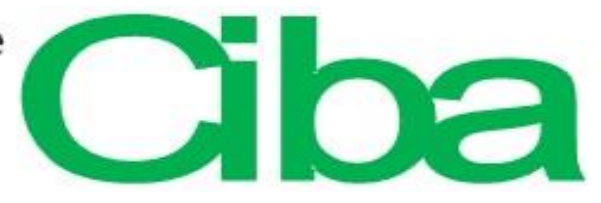

ISSN: 2007 - 9990

2) Validación del modelo del software VENSIM por medio de análisis de regresión con el software SPSS (versión 15).

Se aplicaron modelos de regresión simple por medio del software SPSS (versión 15), para determinar el grado de dependencia entre las variables Producción (ton/ha), en relación con la temperatura $\left({ }^{0} \mathrm{C}\right)$ y la precipitación $(\mathrm{mm})$. Cabe señalar que esto no se hizo con el factor suelo, al no contar con datos de producción completos. A continuación, se presentan los resultados de dichos modelos:

a) Producción de caña de azúcar (ton/ha) y su relación con la precipitación ( $\mathrm{mm}$ ). Con respecto a la producción de caña de azúcar y su dependencia de la precipitación se aplicaron los siguientes modelos de regresión curvilínea: lineal, logarítmico, cuadrático, cúbico y exponencial (Gráfica 2).

Gráfica 2. Análisis de regresión curvilínea para la producción de caña de azúcar y su relación con la precipitación, mediante los modelos lineal, logarítmico, cuadrático, cúbico y exponencial.

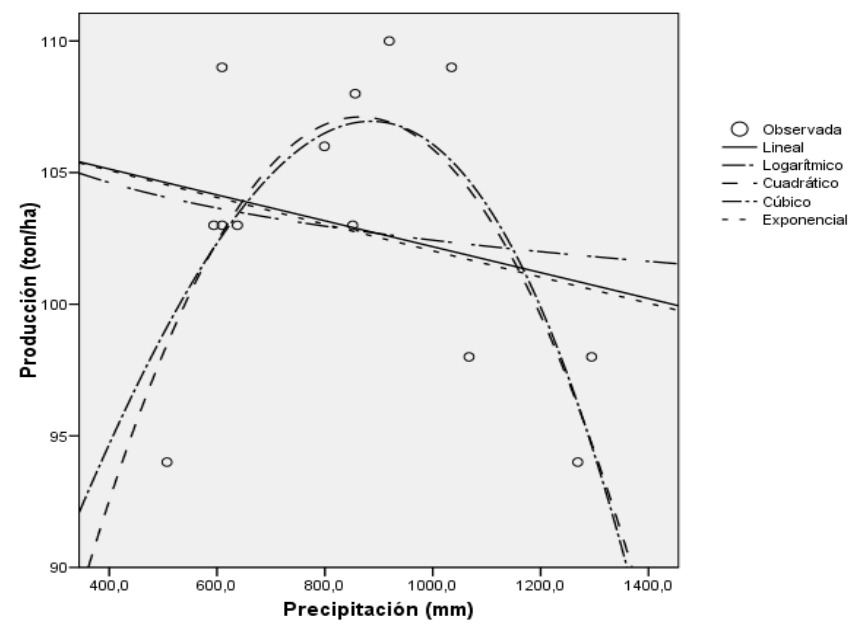




\section{Revista Iberoamericana de las Ciencias Biológicas y Agropecuarias}

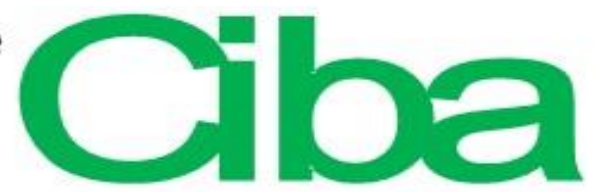

En este caso, el modelo cuadrático y el cúbico fueron estadísticamente significativos (tabla $1)$.

Tabla 1. Resumen del modelo y estimación de los parámetros. Variable Precipitación $(\mathrm{mm})$.

\begin{tabular}{|c|c|c|c|c|c|c|c|c|c|}
\hline \multirow[t]{2}{*}{ Ecuación } & \multicolumn{5}{|c|}{ Resumen del modelo } & \multicolumn{4}{|c|}{ Estimaciones de los parámetros } \\
\hline & $\begin{array}{c}\mathrm{R} \\
\text { cuadrado }\end{array}$ & F & gl1 & $\mathrm{g} 12$ & Sig. & $\begin{array}{c}\text { Constant } \\
\mathrm{e}\end{array}$ & b1 & b2 & b3 \\
\hline Lineal & 0,053 & 0,618 & 1 & 11 & 0,448 & 107,106 & $-0,005$ & & \\
\hline Logarítmica & 0,017 & 0,194 & 1 & 11 & 0,668 & 118,941 & $-2,390$ & & \\
\hline Cuadrático & 0,566 & 6,511 & 2 & 10 & $\underline{0,015}$ & 56,694 & 0,117 & $\begin{array}{c}-6.73 \mathrm{E}- \\
005\end{array}$ & \\
\hline Cúbico & 0,541 & 5,889 & 2 & 10 & $\underline{0,020}$ & 73,644 & 0,056 & 0,000 & $\begin{array}{c}-2.40 \mathrm{E}- \\
008\end{array}$ \\
\hline Exponencial & 0,055 & 0,642 & 1 & 11 & 0,440 & 107,175 & $\begin{array}{c}-4.92 \mathrm{E}- \\
005\end{array}$ & & \\
\hline
\end{tabular}

Variable dependiente: PRODUCCIÓN PROMEDIO

$L$ a variable independiente es $\mathrm{Pp}$.

b) Producción de caña de azúcar (ton/ha) y su relación con la temperatura $\left({ }^{0} \mathrm{C}\right)$. Se estimó la producción de caña y su relación con la temperatura mediante una Regresión curvilínea con los siguientes modelos: lineal, logarítmico, cuadrático, cúbico y exponencial. En todos los casos, no se encontró un modelo de regresión significativo, es decir, no existe dependencia entre la producción de caña de azúcar y la temperatura (tabla 2).

Tabla 2. Resumen del modelo y estimación de los parámetros. Variable Temperatura $\left({ }^{0} \mathrm{C}\right)$.

\begin{tabular}{|c|c|c|c|c|c|c|c|c|c|}
\hline \multirow[t]{2}{*}{ Ecuación } & \multicolumn{5}{|c|}{ Resumen del modelo } & \multicolumn{4}{|c|}{ Estimaciones de los parámetros } \\
\hline & $\begin{array}{c}\mathrm{R} \\
\text { cuadrado }\end{array}$ & $\mathrm{F}$ & gll & $\mathrm{gl} 2$ & Sig. & Constante & b1 & b2 & b3 \\
\hline Lineal & 0,068 & 0,807 & 1 & 11 & $\underline{0,388}$ & 44,910 & 2,641 & & \\
\hline Logarítmica & 0,067 & 0,793 & 1 & 11 & $\overline{0,392}$ & $-74,794$ & 57,526 & & \\
\hline Cuadrático & 0,090 & 0,492 & 2 & 10 & $\overline{0,625}$ & 1146,787 & $-97,684$ & 2,282 & \\
\hline Cúbico & 0,090 & 0,493 & 2 & 10 & $\overline{0,625}$ & 434,269 & 0,000 & $-2,180$ & 0,068 \\
\hline Exponencial & 0,066 & 0,781 & 1 & 11 & $\overline{0,396}$ & 58,632 & 0,026 & & \\
\hline
\end{tabular}

Variable dependiente: PRODUCCIÓN PROMEDIO

La variable independiente es Temp. 
Revista Iberoamericana de las Ciencias Biológicas

y Agropecuarias

\section{Conclusiones}

El comportamiento del suelo será constante para el periodo 2010-2020, y mientras se mantengan las condiciones actuales de las tierras, la producción (ton/ha) se mantendrá con ligeras bajas o elevaciones. Asimismo, cuando los rangos de precipitación se vean alterados, la producción también se verá afectada, generando con ello una disminución de la misma. Respecto a la temperatura, esta no tuvo al menos para este periodo una injerencia muy marcada.

En cuanto a la producción (ton/ha) promedio de la caña de azúcar, se observó con la simulación un ligero aumento en la producción del orden, aproximadamente de 2 toneladas por año, esto es, poco más de veinte toneladas adicionales por hectárea en producción de caña de azúcar para el periodo 2010-2020.

Con respecto a la simulación con el software SPSS, y al estimar la producción de caña y su relación con la precipitación y la temperatura mediante una Regresión curvilínea y con los modelos: lineal, logarítmico, cuadrático, cúbico y exponencial, se coadyuva directamente en la precipitación. Se observó que de todos los modelos de regresión aplicados, solo los modelos cúbico y cuadrático fueron estadísticamente significativos $(p<0.05)$ para la precipitación, probando con ello que sí existe dependencia cúbica y cuadrática entre la producción y dicha variable climática.

Para el caso de la temperatura, todos los modelos de regresión fueron mayores a 0.05 , con lo cual se demuestra con una certeza del $95 \%$ que no existe dependencia entre la producción de caña de azúcar y la temperatura. 
Revista Iberoamericana de las Ciencias Biológicas

y Agropecuarias

\section{Bibliografía}

Acevedo Sandoval O; Valera Pérez, MA; Prieto García, F. 2010. Propiedades físicas, químicas y mineralogía de suelos forestales en Acaxochitlan, Hidalgo, México. Universidad y ciencia 2010. Villahermosa, agosto 2010

Castellanos. J.Z.: Uvalle. B. J.X: Aguilar S.A. (2000). Manual de interpretación de análisis de suelos, aguas agrícolas, plantas y ECP. Segunda edición abril 2000. INCAPA. Guanajuato, México.

CONADESUCA (2015). Comité Nacional para el Desarrollo Sustentable de la Caña de Azúcar. En: http://www.conadesuca.gob.mx/ (consultado el 29 de Octubre de 2015).

CONAGUA. 2014. Precipitación promedio ( $\mathrm{mm}$ ) en el valle de El Grullo-Autlán. Estación “El Chante”. Información de 14 años (2001-2014). Comisión Nacional del Agua.

Ferreras, L.; G. Magra; P. Besson; E. Kovalevski; F. Garcías. 2007. Indicadores de calidad física en suelos de la Región Pampeana Norte de Argentina bajo siembra directa. Ciencia del suelo [online]. 2007, vol.25, n.2, pp. 159-172. ISSN 1850-2067.

García, J. M. 2011. Teoría y ejercicios prácticos de Dinámica de Sistemas. Tercera Edición. Madrid, España. 2011. ISBN 84-607-9304-4.

INEGI. 2000. XII Censo de población y vivienda. Instituto Nacional de Estadística Geografía e Informática. Aguascalientes Ags. Página WEB: http://www.inegi.gob.mx

Keenan C.; Mc Roberts. 2010. Rural development challenges: system dynamics ex ante decision support for agricultural initiatives in southern Mexico. A Thesis. Presented to the Faculty of the Graduate School of Cornell University in Partial Fulfilment of the Requirements for the Degree of Master of Professional Studies International Agriculture and Rural Development

Lesur, L. 2006. Manual de fertilización y productividad del suelo agrícola: una guía paso a paso. México: editorial Trillas. 2006.

Liu, J., Wang, W., Chai, y., \& Liu, Y. 2004. Easy-SC: A Supply Chain Simulation Tool. Proceedings of the 2004 Winter Simulation Conference. New York: IEEE. 
Revista lberoamericana de las Ciencias Biológicas

y Agropecuarias

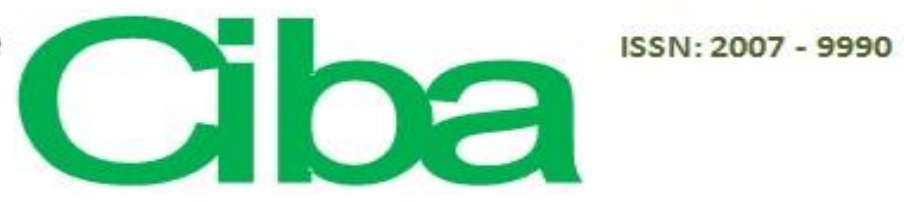

Ortiz V., B. y C. A., Ortiz S. 1990. Edafología. $7^{a}$ Edición. Editora V. A. Universidad Autónoma de Chapingo. Texcoco, Estado de México.

SEI-JAL. 2000. Sistema Estatal de Información Jalisco, en base a datos proporcionados por INEGI. Jalisco. Resultados Definitivos, Datos por Localidad (Integración Territorial, X1 Censo General de Población y Vivienda, 1990. CGSNEGI.

SPP. 1981. Síntesis de Información Geográfica de Jalisco. Secretaría de Programación y Presupuesto.

SPSS. (Software. Version 15,).

USDA. 2011. Word production, supply, and distribution, centrifugal sugar (table 1). Sugar and Sweetener Yearbook, 2011. United States of America Department Agricultural.

UNC. 2011). México en el mundo. Unión Nacional de Cañeros, A. C.-CNPR. En http://www.caneros.org.mx/principal.html\#. 